\title{
Development of Cheese Rectangular Coordinate Robot Control System
}

\author{
Nie Jungang \\ Advanced Manufacture Technology Center of China \\ Academy of Machinery Science Technology . \\ Bei Jing 100044. \\ e-mail: nie_jg@163.com
}

\author{
Ren Yongxin, Chi Changhao, Zhang Jing \\ Advanced Manufacture Technology Center of China \\ Academy of Machinery Science Technology . \\ Bei Jing 100044. \\ e-mail: nie_jg@163.com
}

\begin{abstract}
There are labor intensive, harsh environment and other issues in the handling process of the cheese in the dyeing workshop. To solve this problem we have designed and developed a Cheese Rectangular Coordinate Robot. PC is the host computer, and the software in PC is compiled in VC environment. It responsibles for control management and data management. Motion controller is slave computer, it drives servo motors and ancillary control equipment to achieve the function of robots picking and placing the yarns. The production practice approves that this robot control system is able to communicate with central control system and control robots' motion, and the system is stable and reliable.
\end{abstract}

Keywords-Rectangular Coordinate;Robo control; Database; Visual $C++$

\section{INTRODUCTION}

In China's textile printing and dyeing industry, Loading and unloading of yarn equipment's automation level is low[1,2], and in cheese dyeing automation production line Mostly adopt the semi-automatic production mode, Through artificial complete bobbin transfer between each workstation in production line. But great changes have taken place in China's population structure at present, facing the grim problem of labor shortage, artificial cost gradually increase. As a laborintensive and bad environment industry, textile industry also faced a severe test of the "labor shortage". In order to solve the problems of the "labor shortage" and upgrade the industrial structure, we develop a cheese rectangular coordinate robot control based on VC and PMAC. The robot uses he IPC + PMAC structure, and In Visual c + + environment using object-oriented method to develop the control software of the robot. This control system Realize the information interaction with the central control system and other related equipment.

\section{CATEGORY RECTANGULAR COORDINATES RoBOT}

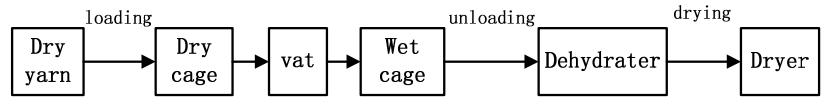

Figure 1. Cheese yarn dyeing logistics map

In the textile dyeing and finishing production line, cheese production and logistics: dry cheese yarn is loaded to cage pole, each pole mounted ten yarn, the number of cage pole is different depends on the model and size; the cage with dry cheese yarn will be equipped into the vat by crane to be dyed; After staining is completed, the cage with wet yarn will be equipped hanged on unloading station; after Dehydration, the bobbin is transported to the dryer for drying. Specific logistics process shown in Figure 1.

In the logistics process, the loading, unloading and drying of yarn aspects labor-intensive, and will be come into contact with the dye wash, so we developed rectangular coordinate robots: loading robot, unloading robot and drying robot. The loading and the unloading robot are four-axis robot, the drying robot is three-axis robot, but All three machines use the same design principles and techniques, just grab yarn with different clutch according to different working conditions.

\section{ROBOT CONTROL SYSTEM's HARDWARE}

The Cheese Rectangular Coordinate Robot control system is a kind of typical multi-axis real-time control system, using two stage control system, motion control card is used as lower computer, and Industrial PC is used as upper computer. It is communicated with the lower actuator in real time, Can rapidly and accurately control motor unit's movement, and also can Real-time control the auxiliary system through the switch circuit control system. Industrial PC has the advantages of fast speed, large storage capacity, flexible application ${ }^{[3,4]}$, so Can be used in large scale computation and complex task management. The control system based on motion control card and industrial PC, fully consider their strengths, improve the overall performance of the system ${ }^{[5-7]}$. The robot control system's hardware is illustrated in Figure. 2. 


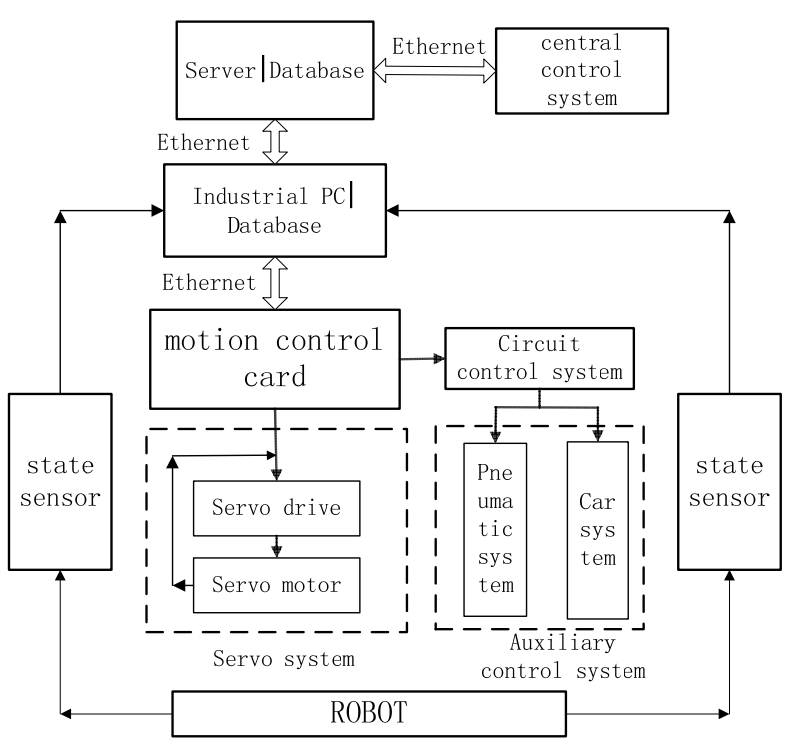

Figure 2. Robot control system's hardware

The robot motion structure mainly includes: the lateral motion of the $\mathrm{X}$ axis, transverse motion of $\mathrm{Y}$ axis, the longitudinal motion of $\mathrm{Z}$ axis, the Rotary motion of $\mathrm{A}$ axis, and the linkage motion of $\mathrm{X}$ axis, $\mathrm{Y}$ axis, $\mathrm{Z}$ axis, $\mathrm{A}$ axis, the Pneumatic control system, The car movement control system etc. This robot is a part of the cheese dyeing automation production line, In actual production application, the Central control system according to the operation state of the whole production line equipment and material, to send out scheduling Instruction to the system scheduling database in server. Industrial PC is respectively connected with the server and the control card through Ethernet, the Control management system software loop to read the scheduling Instruction of the system scheduling database in server, and sent out the execution instruction to motion control card, At the same time it gets running status of the control card and the auxiliary system, and stores the status into the database in Industrial PC. on the one hand, control card is connected with industrial PC through the network port, downloads the rectangular coordinate robot trajectory data, parameters and instruction which is processed by control and management system software, and process these data through the unit of DSP; On the other hand, control card is connected with Servo drive, output PWM digital signal, to control the servo motor to do motion, thus to complete the robot's action include getting bobbin, catching bobbin and transport etc. control card is also connected with the Auxiliary control system through Circuit control system to Realize control them.

\section{THE STRUCTURE OF ROBOT CONTROL SYSTEM SOFTWARE}

From the control strategies considered, the control system software includes three parts: Real time control part, it is the motor program, includes motion control card and industrial PC, it's mainly responsible for the built-in PLC program running, interpolation and servo control; The communication driver between the upper and lower computer; The control and management system software, which is installed in the industrial PC.
The system software structure diagram is illustrated in Figure. 3.

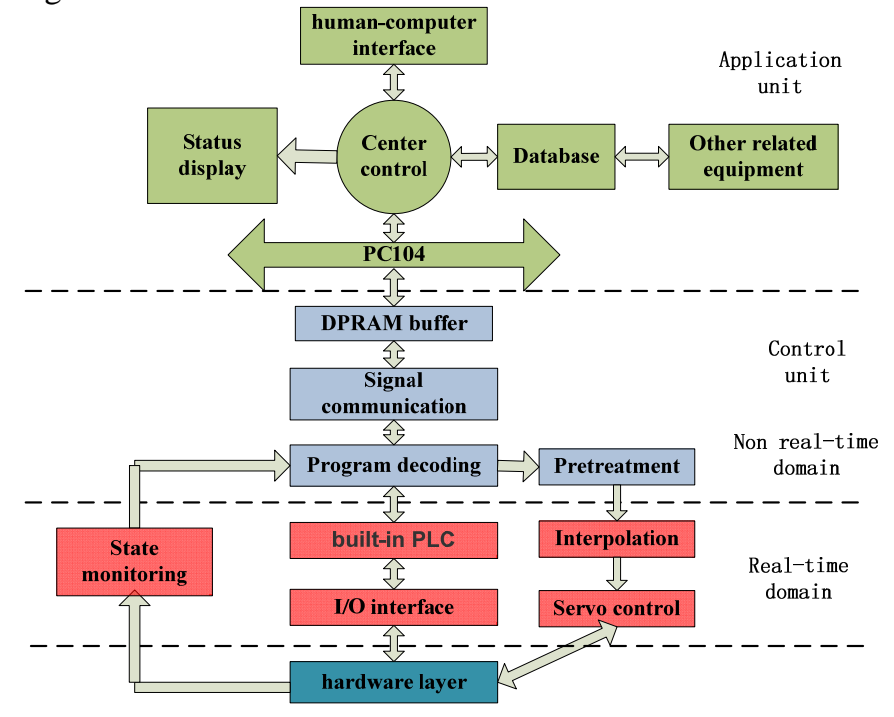

Figure 3. system software structure diagram

\section{A. The Real-time Control Motion Program Modular}

The motion control card can support up to 256 motion program[7,8], Each motion program to control the robot's a motion sequence or motion state, through the motion program can decompose the robot's motion into multiple independent motion segment, so the control software is easy to control and call. The motion program is divided into two types, built-in PLC program and movement execution program. The built-in PLC programs are mainly used for monitoring robot's various sensors, a continuous cycle of scans for the sensors to get their states, to execute corresponding operation according to the different state of sensors. The movement execution programs are used for each motion axis' motion control. Writing a movement execution program, first need to define each axis' coordinate system, In the control card coordinate system refers to one or a group organized motors which for synchronization purposes, establishing coordinate system for each motor through definition statement $[9,10]$. Secondly Defining the motion parameters and status parameters, and according to the desired trajectories and control actions using control card programming grammar writing code, download to the control memory. The final acceptance control management software's instruction and coordinate parameters value, perform motion program, to allow the robot to complete the corresponding action.

\section{B. The Control and Management System Software}

The upper computer using generic Windows operating system, SQL2005 database, and designing the control and management system software with $\mathrm{VC}++6.0$. Microsoft SQL Server 2005 is a comprehensive database platform, it uses integrated business intelligence (BI) tool, provides more secure and reliable storage function for relational data and structured data, and it can construct and management Applications program for Processing information data. Visual $\mathrm{c}++6.0$ encapsulates the Windows API function library, forming a MFC (Foundation Class Microsoft) class library, and MFC class 
libraries can be used to quickly and easily develop applications for the requirements. In order to make the robot control system software, easy to maintain, with reconfigure ability and extensibility, we design the software with the modular method. The software is divided into the following modules: manual module, Single step operation module, Single / online control module, Coordinate display module, monitoring module.

The specific functions of each module are as follows:

Manual module: the manual operation including $\mathrm{X}$ axis', $\mathrm{Y}$ axis', $\mathrm{Z}$ axis', A axis' manual operation of mobile, the robot's return to zero etc..Single step operation module: it's Mainly used for manual control of the robot's ancillary equipment, such as the Mechanical hand, the central guide rod, and the cylinder etc..Single / online control module: Can choose the Single or online mode. In the single state, you can manually control the operation of the robot; in the online state, the central control system issue task orders to robot, according to the orders and itself state, the robot automatically perform the grasping and unloading action. Coordinate display module: Display the current location of each coordinate axis of the robot. monitoring module: monitoring the connection status with database, whether motor program is fully implemented, and whether the robot's axis is in the zero position etc..

\section{The Limit of Eeach Axis of The Robot}

Robot needs not only multi axis motion control card to control the movement, and will need to be added the limit switch to constrain its motion, To prevent the robot from crossing the limit position to cause dangerous accidents, Thus constitutes a closed-loop control system. In order to solve this situation, design the three layers of security protection measures:

First, the soft limit is arranged in each axis of multi axis motion control card. There are two Soft limit variables which are Mxx31 and Mxx32, "XX" means the motor axis, Mxx31 is a positive limit setting, and Mxx32 is a negative limit setting. When the negative limit is set, must add the "-". For example, the positive limit of the 1 axis is M131, $\mathrm{M} 131=40$ 000; the negative limit of the 1 axis is M132, which is M132=-40 000 .

Second, access limit switch. The robot arm is not easy to install the travel switch, so you can choose to install the proximity switch. The proximity switch is small, easy to install and sensitive. It can be divided into normally closed and normally open. If adopt the normally closed switch, when the axial movement did not reach the limit position, the proximity switch's Indicator light has been in a state of light; When the axial movement to the limit position (to the proximity switch induction zone), the proximity switch receives the sensing signal, indicating lights put out, At the same time, the signal feedback to the upper computer, So the upper computer quickly respond to send commands to the corresponding motor stop motion.

Finally, use the movement control card's built-in PLC program to continuously scan the position of the axis, to prevent the occurrence of accidents due to the failure of the limit switch. Since the robot uses an incremental encoder, the robot needs to get back to the zero position when the robot starts to work, and establish the initial coordinate system. When back to zero position, if the proximity switch failure, an accident may occur. Write a built-in PLC procedures, and constantly detect whether each axis normal return zero position. If back to zero position is not normal (proximity switch failure), PLC program did not detect the signal of back to zero position, it will send to stop the axis motor sports instruction, it can ensure that the shaft is not beyond the limit position. When the proximity switch fails, the built-in PLC program will send commands to the upper computer, so that all the motor to stop the movement in order to artificial maintenance, to avoid accidents.

\section{CONCLUSION}

This paper describes a kind of cheese rectangular coordinate robot control system based on VC and motion control card, this system adopts the Hierarchical control strategy which include industrial PC as upper computer and control card as lower computer, control card is responsible for real-time motion control program, IPC is responsible for control and management system software, between upper and lower computer communicate through PComm32 driver. At the same time, the robot control system and cheese dyeing automation production control system for information interaction through the database. Through production practice verification, the running state of the robot is good, it improves production efficiency, greatly reduces the labor intensity, reduces the quantity of the employment, bring economic benefits for enterprises.

\section{ACKNOWLEDGMENT}

This work was financially supported by special funds o f Research Institute of technology research and developme nt (2013EG119164).

\section{REFERENCES}

[1] Kim Y H, Huff-Lonergan E, Sebranek J G, et al. High-oxygen modified atmosphere packaging system induces lipid and myoglobin oxidation and protein polymerization [J], Meat science, 2010, 85(4): 759-767.

[2] Kim Y H, Huff-Lonergan E, Sebranek J G, et al. High-oxygen modified atmosphere packaging system induces lipid and myoglobin oxidation and protein polymerization [J], Meat science, 2010, 85(4): 759-767.

[3] Dou Wei, Zhao Xinhua, Li Zhenhua. Development of Robot Control Software Base on $\mathrm{VC}++$ and PMAC[J]. Journal of Tianjin University of Technology, 2010, 26(3): 58-61.

[4] Peng Shaobo, Zhou Jian, Li Lijun, Yi Chunfeng, Gao Zicheng, Zhang Xiang. Motion Control of Picking Robot Based on PM AC [J]. Journal of Agricultural Mechanization Research, 2014, 2: 7678.

[5] Zhang Hong, Xiong Wenlong. C /S Based Network Usage of SQL Database Technology[J]. Journal o f Wuhan University of Techno logy( Transportation Science\& Engineering ), 2003, 27(6): 890-892.

[6] Lan Guangming, Han Qiushi, Sun Zhiyong. Real Time Data Acquisition and Display on PMAC Under The Environment of Visual $\mathrm{C}++[\mathrm{J}]$. Journal of Beijing Institute of Machinery, 2004, 19(1): 61-65.

[7] Delta Tau Data System Inc . PMAC Pewin32PRO SOFTWAREREFERENCE [M] . USA: DELTA TAU Data System Inc, 1999. 
[8] Lan Wenbao. Research and Implementation of PMAC-based Industrial Robot Control System[D]. Harbin: Harbin Engineering University, 2012.
[9] LIU L, SHELL D A. Assessing optimal assignment under uncertainty: an interval-based algorithm[J]. Intl J Robotic Res, 2011,30( 7) : 936-953.

[10] John J Craig. Introduction to robotics mechanics\& control [M ] New York : Addison-Wesley Publishing Company,1986. 